This item was submitted to Loughborough's Research Repository by the author.

Items in Figshare are protected by copyright, with all rights reserved, unless otherwise indicated.

\title{
Microneurographic characterization of sympathetic responses during 1-leg exercise in young and middle-aged humans
}

\section{PLEASE CITE THE PUBLISHED VERSION}

https://doi.org/10.1139/apnm-2018-0101

\section{PUBLISHER}

(C) NRC Research Press

\section{VERSION}

AM (Accepted Manuscript)

\section{PUBLISHER STATEMENT}

This work is made available according to the conditions of the Creative Commons Attribution-NonCommercialNoDerivatives 4.0 International (CC BY-NC-ND 4.0) licence. Full details of this licence are available at: https://creativecommons.org/licenses/by-nc-nd/4.0/

\section{LICENCE}

CC BY-NC-ND 4.0

\section{REPOSITORY RECORD}

Notarius, Catherine F., Philip J. Millar, Connor J. Doherty, Anthony V. Incognito, Nobuhiko Haruki, Emma ODonnell, and John S. Floras. 2019. "Microneurographic Characterization of Sympathetic Responses During 1-leg Exercise in Young and Middle-aged Humans". figshare. https://hdl.handle.net/2134/34426. 


\title{
MICRONEUROGRAPHIC CHARACTERIZATION OF SYMPATHETIC RESPONSES DURING 1-LEG EXERCISE IN YOUNG AND MIDDLE-AGED HUMANS
}

\author{
Catherine F. Notarius ${ }^{1}$, Philip J. Millar ${ }^{1,2}$, Connor J. Doherty ${ }^{2}$, Anthony V. Incognito ${ }^{2}$, \\ Nobuhiko Haruki ${ }^{1}$, Emma O’Donnell ${ }^{1,3}$, John S. Floras ${ }^{1}$
}

\begin{abstract}
${ }^{1}$ University Health Network and Mount Sinai Hospital Division of Cardiology, University of Toronto. Address: 6ES:414, Toronto General Hospital, University Health Network, 200 Elizabeth St. Toronto, ON, Canada,M5G 2C4. Email: c.notarius@utoronto.ca; harukinobuhiko0705@gmail.com; john.floras@utoronto.ca

${ }^{2}$ Dept. of Human Health and Nutritional Sciences, University of Guelph, 341A ANNU, 50 Stone Road East, Guelph ON, Canada, N1G 2W1. Email: pmillar@uoguelph.ca; cdoher02@uoguelph.ca; aincogni@uoguelph.ca

${ }^{3}$ School of Sport, Exercise and Health Sciences, Loughborough University, United Kingdom. Email: e.odonnell@lboro.ac.uk
\end{abstract}

Short Title: Aging and sympathetic response to exercise

Corresponding author: C.F. Notarius Ph.D., 6ES:414, Toronto General Hospital, University Health Network, 200 Elizabeth St. Toronto, Ontario; Canada, M5G 2C4

Tel: (416) 340-4755; Fax: (416) 340-5506; email: c.notarius@utoronto.ca 


\begin{abstract}
Muscle sympathetic nerve activity (MSNA) at rest increases with age. However, the influence of age on MSNA recorded during dynamic leg exercise is unknown. We tested the hypothesis that aging attenuates the sympatho-inhibitory response observed in young subjects performing mild to moderate 1-leg cycling. After pre-determining peak oxygen uptake (VO2peak), we compared contra-lateral fibular nerve MSNA during 2 minutes each of mild (unloaded) and moderate (30-40\% of the work rate at peak $\mathrm{VO}_{2}$, halved for single leg) 1-leg cycling in 18 young $(23 \pm 1$ years $[$ mean \pm SE] $)$ and 18 middle-aged ( $57 \pm 2$ years $)$ sex-matched healthy subjects. Mean height, weight, resting heart rate (HR), systolic blood pressure (BP) and percent predicted $\mathrm{VO}_{2}$ peak were similar between groups. Middle-aged subjects had higher resting MSNA burst frequency and incidence $(\mathrm{P}<0.001)$ and diastolic $\mathrm{BP}(\mathrm{P}=0.04)$. During moderate 1-leg cycling, older subjects' systolic BP increased more $(+21 \pm 5$ vs. $+10 \pm 1$ $\mathrm{mmHg} ; \mathrm{P}=0.02)$ and their fall in MSNA burst incidence was amplified $(-19 \pm 2$ vs. $-11 \pm 2$ bursts/100heartbeats; $\mathrm{P}=0.01)$ but because HR rose less $(+15 \pm 3$ vs. $+19 \pm 2 \mathrm{bpm} ; \mathrm{P}=0.03)$, exercise induced similar reductions in burst frequency $(\mathrm{P}=0.25)$. Contrary to our initial hypothesis, with advancing age, mild to moderate intensity dynamic leg exercise elicits a greater rise in systolic BP and a larger fall in MSNA.
\end{abstract}

Keywords: aging, microneurography, dynamic exercise 


\section{INTRODUCTION}

Human aging is accompanied by an increase in central sympathetic outflow to skeletal muscle (muscle sympathetic nerve activity; MSNA) (Sundlof \& Wallin, 1978) without any concurrent diminution of its arterial baroflex modulation (Ebert et al., 1992;Rudas et al., 1999; Matsukawa et al., 1996). This increased neural vasoconstrictor drive is paralleled by reductions in resting muscle blood flow, vascular conductance (Dinenno et al., 2001; Hart et al., 2009), arterial compliance (Tanaka et al., 2000), and higher systolic blood pressure (Franklin et al., 1997).

Less is known about the effects of healthy aging on MSNA during exercise,which engages simultaneously autonomic reflexes elicited by mechanically- and metabolicallysensitive group III/IV skeletal muscle afferents (exercise pressor reflex), stretch sensitive baroreflexes, and feed-forward signals from higher brainstem regions (central command) (Crisafulli et al., 2015). The firing of single sympathetic units engaged by these stimuli integrate to comprise the multi-fiber neurogram recorded by microneurography (Valbo et al., 2004).

To date, most exercise studies involving MSNA have employed static handgrip (HG) protocols. These investigations have not yielded consistent results: age either attenuated (Houssiere et al., 2006) or had no effect (Ng et al., 1994;Markel et al., 2003; Greaney et al., 2013;Lalande et al., 2014) on the reflex increase in MSNA elicited by this stimulus. Leg exercise at mild to moderate intensities is more representative of daily human activity than static HG. Whereas moderate intensity static HG increases MSNA in young healthy subjects, principally by stimulating group III/IV skeletal muscle afferents sensitive to metabolic stimuli (muscle metaboreflex) (Mark et al., 1985;Victor et al., 1987;Saito \& Mano, 1991), dynamic leg exercise of comparable intensity decreases MSNA (Saito et al., 1993;Ichinose et al., 2008;Callister et al., 1994;Katayama et al., 2011;Doherty et al., 2018). 
This latter observation has been attributed to concurrent engagement of the skeletal muscle pump to increase cardiac filling pressure; the consequent stimulation of cardiopulmonary baroreceptors will inhibit reflexively central sympathetic outflow (Ray et al., 1993;Katayama et al., 2014). Whether 1-leg exercise elicits similar sympatho-inhibition in middle-aged subjects has not been reported.

Prior studies of aging in which cardiopulmonary mechanoreceptors were unloaded by lowering cardiac filling pressures reported either an augmented (Davy et al., 1998;Davy et al., 1998) or unaltered (Tanaka et al., 1999) increase in MSNA. Conversely, dynamic exercise should stimulate these baroreceptors, decreasing MSNA reflexively. However, several age-related factors could modify the influence of the skeletal muscle pump on the autonomic adjutments to exercise (Proctor D.N. \& Parker, 2006), including a decrease in muscle mass (Frontera et al., 2000), a change in total blood volume (Davy \& Seals, 1994), and/or changes in venous compliance (Olsen \& Lanne, 1998).

The principal aim of the present study was to compare, in young and healthy middleaged subjects, the magnitude of sympatho-inhibition evoked by short duration low intensity dynamic 1-leg cycling. We hypothesized that healthy aging attenuates the fall in multi-unit MSNA observed in young subjects over the first 4 minutes of such exercise.

\section{METHODS}

\section{Subjects}

Thirty-six healthy, medication-free, volunteers were recruited through local advertisement, screened by medical history, and divided into 2 groups: 18 young (mean age $23 \pm 1$ years; range 18-28; 6 women) and 18 middle-aged (mean age $57 \pm 2$ years; range $48-72 ; 6$ women). By definition, all were normotensive and in sinus rhythm; none was diabetic or obese. This protocol represents one element of a larger study approved by the Research Ethics 
Boards at both University Health Network (Reference number 09-0988-AE), Mount Sinai Hospital (10-0013-E), and the University of Guelph (15JN004). Informed written consent was obtained from all subjects.

\section{Procedures}

Subjects were studied in a quiet temperature-controlled laboratory at the same time of day, two hours following the last food intake, on two separate days. All participants abstained from caffeine as well as vigorous exercise for 12 hours beforehand.

On the first day, oxygen uptake at peak exercise (VO2peak) was determined by open circuit spirometry (Quark CPET system, Cosmed USA Inc., Chicago, IL or Moxus Modular VO2 System, AEI Technologies, Pittsburgh PA). Subjects performed a graded ramped bicycle ergometer test (17-30 watts/min) with both legs until pedal speed could not be maintained above $50 \mathrm{rpm}$ and the respiratory exchange ratio $\left(\mathrm{VCO}_{2} / \mathrm{VO}_{2}\right)$ exceeded 1.1. Work increments were individualized, based on physical activity status, to ensure that exercise duration would not exceed 14 minutes. VO2peak was expressed both as $\mathrm{ml} / \mathrm{kg} \cdot \mathrm{min}^{-1}$ and as percent of predicted VO2 peak, accounting for age, sex, body weight and height (Jones et al., 1985). The work rate at 30-40 percent of that at VO2peak was based on 2-legged cycling. This value was halved in order to determine an equivalent moderate intensity work rate for the 1-leg cycling protocol. Therefore the moderate work rate for 1-leg was approximately 15$20 \%$ of the peak work rate at VO2peak.

On the second day subjects sat in a comfortable chair with the left leg supported on a stool and the right leg secured to the pedal of a floor-mounted cycle ergometer (Monark Rehab Ergometer Trainer 881, Sweden). Right arm blood pressure was recorded automatically each minute (Dinamap Pro 100, Critikon, Tampa, FL) at rest and during exercise. In 10 of the young subjects, blood pressure during exercise was assessed during exercise by calculating 30 
second averages of beat-by-beat blood pressure (Finometer MIDI, Finapres Medical Systems, The Netherlands), calibrated at rest (BPtru Model BPM-200, BpTRU, Coquitlam, BC). Heart rate was derived from lead II of an electrocardiogram. A respiratory belt encircled the abdomen. Multiunit recordings of post-ganglionic MSNA were obtained with a unipolar tungsten electrode inserted selectively into a left peroneal (fibular) muscle-nerve fascicle, as previously described (Notarius et al., 2015). To compare between-group differences in exercise-induced reflex and central effects on efferent sympathetic nerve discharge (the principal variable of interest), MSNA was expressed as firing incidence (bursts/100 heartbeats). MSNA also was expressed as firing frequency (bursts/min), a measure that provides insight into the potential functional importance of changes in central sympathetic outflow for neural norepinephrine release and vascular resistance (Valbo et al., 2004). MSNA was analyzed using a custom semi-analytic program constructed on LabVIEW ${ }^{\circledR}$ (National Instruments, Austin, Texas) (Notarius et al., 2015).

\section{Protocol}

After 10 minutes of quiet rest, baseline signals were acquired over 7 minutes of spontaneous breathing. MSNA was recorded from the left leg at rest and during early onset, non-steady state 1-leg cycling (right leg) for 4 minutes ( 2 minutes at zero load and 2 minutes at $15-20 \%$ of the work rate at peak $\mathrm{VO}_{2}$ ). Although the target intensity was 20 percent of the work rate at peak $\mathrm{VO} 2$, a slight reduction was required in 8 participants $(3$ older and 5 younger) who could not keep the stationary leg still when the absolute work rate was highest. Subjects pedalled at 60-70 rpm, Zero load refers to short duration unloaded cycling, i.e. no added resistance. After 2 minutes of unloaded 1 leg cycling a resistance comparable to 15$20 \%$ of the measured work rate at peak VO2 was applied for a further 2 minutes. At both work rates, subjects rated their perceived exertion (RPE) according to the modified Borg scale (0-10) (Noble et al., 1983). 


\section{Statistical Analysis}

Data are presented as mean \pm standard error. Depending on the distribution of data, we compared differences between group means by unpaired t-tests or Mann-Whitney Rank sum tests. We compared absolute changes from baseline in dependent variables during the second minute of unloaded and loaded dynamic 1-leg cycling between the middle-aged and younger groups, by applying a two-factor repeated measures analysis of variance (ANOVA)(SigmaStat ${ }^{\mathrm{TM}}$ for Windows, Ver. 3.5, Systat Software Inc., Chicago, IL), with group (middle-aged vs. younger) and exercise intensity (mild and moderate work rate) as the two factors. A post-hoc Student Newman-Keuls test assessed individual differences between means. Pearson correlation analysis was applied to test for an association between peak oxygen uptake and MSNA.

\section{RESULTS}

Physical characteristics and baseline measures

Summary data appear in Table 1. Of note, the groups exhibited similar mean height, weight, resting heart rate, and systolic blood pressure (all $\mathrm{P}>0.05$ ). Mean resting diastolic blood pressure was higher in the older cohort $(\mathrm{P}=0.04)$, as were resting MSNA burst frequency and burst incidence (both $\mathrm{P}<0.001)$. If expressed in absolute terms $(\mathrm{P}=0.004)$ or relative to body mass $(\mathrm{P}<0.001)$, peak $\mathrm{VO}_{2}$ was significantly less in the middle-aged group. However, there was no between-group difference when calculated as a percent of predicted $\mathrm{VO}_{2}$ peak $(\mathrm{P}=0.52)$, indicating that, relative to age, the exercise capacity of both groups was matched. As expected, there was no relationship between resting MSNA and VO2peak in either age group nor in the group as a whole.

\section{Cycling Exercise}


Blood pressure was not available in 6 of 18 middle-aged subjects during moderate exercise because of cuff malfunction due to increased muscle tension in the arm. The mean change in systolic blood pressure during moderate cycling was significantly greater in the middle-aged subjects $(+21 \pm 5$ vs. $+10 \pm 1 \mathrm{mmHg}, \mathrm{P}=0.02)$ whereas diastolic blood pressure did not differ $(\mathrm{P}=0.22)$ (Figure 1). At the moderate work rate, the rating of perceived exertion (RPE) tended to be higher in the middle-aged group ( $3.9 \pm 0.4$ vs. $2.8 \pm 0.3, \mathrm{P}=0.05)$, although their absolute work rate was lower $(23 \pm 2$ in middle-aged vs $30 \pm 2$ watts in the young, $\mathrm{P}=0.04)$. Both exercise intensities elicited a fall in MSNA burst incidence; this reduction was greater in the middle-aged compared with younger cohort in response to both mild (-14 \pm 2 vs. $-9 \pm 2, \mathrm{P}<0.05)$ and moderate $(-19 \pm 2$ vs. $-11 \pm 2$ bursts/100 heartbeats, $\mathrm{P}=0.01) 1$-leg cycling (Figure 2A).

In both groups, heart rate increased from baseline during both levels of exercise (main effect of time $\mathrm{P}<0.001$ ) but less in middle-aged subjects (interaction $\mathrm{P}=0.03$ ). Consequently, at each work rate there were similar reductions in the average MSNA burst frequency of young and middle-aged subjects $(\mathrm{P}=0.25)$ (Figure $2 \mathrm{~B})$.

\section{DISCUSSION}

The aim of this study was to compare the MSNA responses of healthy young and middle-aged, individuals to short duration mild and moderate intensity dynamic leg exercise. At rest MSNA burst frequency and incidence were higher in the middle-aged participants and similar to values previously published (Notarius et al., 2015) (Millar et al., 2015) (Davy et al., 1998;Houssiere et al., 2006;Hart et al., 2015). Our hypothesis was that aging would attenuate the contralateral sympatho-inhibitory response observed when young subjects perform mild to moderate 1-leg cycling. However, we observed the opposite response during both mild and moderate dynamic leg cycling: for a similar relative workload and a trend 
towards a higher perceived exertion, the fall in MSNA burst incidence, representing central sympathetic outflow, was greater in the older group. As anticipated from prior literature describing age-related decreases in chronotropic reserve (Fleg et al., 1994;Correia et al., 2002;Ferrari et al., 2003;Fisher et al., 2010), heart rate increases during cycling were blunted in our middle-aged subjects. As a consequence, reductions in the frequency of nerve firing were similar in the two groups.

Ray and colleagues (Ray et al., 1993) were the first to report a decrease in MSNA burst frequency (fibular nerve) during mild and moderate dynamic leg extension in young healthy seated subjects. Because this response was absent when exercise was performed supine, the sympatho-inhibition observed was attributed to loading of the cardiopulmonary baroreflex by muscle pump-induced elevations in central venous pressure during upright rhythmic leg exercise (Ray et al., 1993). That initial observation was subsequently confirmed but restricted to low work rates only (10-20 watts) and documented also in the median or radial nerve during leg cycling (Saito et al., 1993;Ichinose et al., 2008;Callister et al., 1994;Katayama et al., 2011). However, at higher exercise intensities ( $>50-60 \%$ of VO2 peak and continued to exhaustion), MSNA rose rather than fell (Ray et al., 1993;Katayama et al., 2011; Ichinose et al., 2008).

In the present series, the novel age-related effect on the central sympathetic response elicited by the onset of mild and moderate dynamic leg exercise invites speculation as to potential causal mechanisms. Since perceived exertion tended to be greater in middle-aged subjects, it is more likely that the greater reduction in burst incidence observed reflects agerelated differences in autonomic reflex input and central network processing rather than attenuated central command.

Arterial baroreflex modulation of heart rate is impaired in older humans (Monahan, 2007), but its control of sympathetic outflow to skeletal muscle vasculature is preserved 
(Ebert et al., 1992;Rudas et al., 1999;Matsukawa et al., 1996;Monahan, 2007). As anticipated from the literature, the systolic blood pressure response to moderate cycling in the middleaged group was double that of younger participants (Fleg et al., 1994;Correia et al., 2002; Ferrari et al., 2003;Fisher et al., 2010). Greater arterial stiffness with increasing age may have contributed to this effect (Redfield et al., 2005). Interestingly, Studinger et. al. observed, in older subjects, an accentuated sympathoinhibitory response to an arterial pressure rise (Studinger et al., 2009). Thus, one plausible interpretation of the present findings is that the augmented sympatho-inhibition of older subjects reflects primarily an appropriate and proportional arterial baroreceptor reflex response commensurate with this 2fold greater rise in systolic pressure.

Interactions with sympatho-inhibitory cardiopulmonary baroreceptor reflexes, stimulated by muscle-pump induced increases in preload also should be considered. When studied, in isolation, using head down tilt, the reflex sympatho-inhibitory response to an increase in central venous pressure appears preserved with age (Tanaka et al., 1999). However, the same group also reported, with aging, an augmented reflex sympathetic excitatory response to combined unloading of low and high pressure baroreceptors via graded lower body negative pressure (they did not evaluate the inhibitory response to concurrent increases in cardiac filling and systemic pressures) (Davy et al., 1998). In an earlier comparison of young (aged 18 to 36), and older subjects (aged 60 to 69), in which reflex increases in sympathetic outflow were inferred from the magnitude of forearm vasoconstriction in responses to both selective and combined unloading of carotid and cardiopulmonary mechanoreceptors, the slope of the relationship between changes in forearm vascular resistance and central venous pressure was similar in young and older subjects but the additive summation of the two responses observed in young participants was absent in the older group (Shi et al., 1996). From the latter finding it was concluded that aging alters the 
central integration of afferent neural input from these mehanoreceptor populations.

Furthermore, it has been argued that the muscle pump may be less effective in raising preload in older than in younger individuals (Proctor D.N. \& Parker, 2006). Thus, when such evidence is considered in aggregate, the anticipated net response, in the context of the present protocol, would be an attenuated, rather than augmented central sympathetic response to exercise.

The present levels and duration of 1-leg cycling are unlikely to activate the muscle metaboreflex in young healthy subejcts, but whether this also is the case for middle-aged adults is uncertain as the literature concerning aging and the muscle metaboreflex is inconsistent. Some investigators report preserved muscle metaboreflex responses with age (Ng et al., 1994;Greaney et al., 2013;Sidhu et al., 2015), whereas others have observed an impaired MSNA reflex response (Houssiere et al., 2006; Markel et al., 2003). Importantly, in the context of the present discussion, is the observation of preserved interaction, with age, between the arterial baroreflex and the muscle metaboreflex regulation of sympathetic outflow (Ng et al., 1994; Greaney et al., 2013).

\section{Limitations}

The World Health Organization defines aging for statistical purposes as 60 years and older, which is slightly higher than the mean age of our middle-aged cohort (World Health Organization, 2013). Subjects in the present study were matched for relative $\mathrm{VO}_{2}$ peak, expressed as the percent of predicted value based on age and body size and of note, these were above population averages. Thus, relative fitness levels were similar and would not explain the observed difference in sympathetic responses. These results are limited to the mild and moderate work rates studied and may not be representative of higher exercise intensities. 
The principal limitation to the interpretation of this study's key finding is the absence of data informing mechanisms. In particular, the effects of the present exercise on cardiac preload in the young and older participants was not determined.

\section{Summary}

The present study is the first to directly examine differences in the contralateral muscle sympathetic response to early onset, non-steady state dynamic leg exercise in middleaged and young subjects. Contrary to our initial hypothesis, the drop in MSNA burst incidence during short duration mild and moderate intensity cycling was augmented in the older participants. We attribute this finding to an age-related effect on autonomic reflexes engaged by exercise and in particular amplified arterial baroreflex-mediated sympathoinhibition, induced by the 2-fold greater increase in systolic blood pressure elicited when older subjects exercise. 


\section{ACKNOWLEDGEMENTS}

We acknowledge with thanks the technical and administrative support of Beverley Morris RN.

\section{GRANTS}

This study was supported by operating grants from the Heart and Stroke Foundation of Ontario (T4938, NA6298), the Canadian Institutes of Health Research (PJT148836), and the Natural Science and Engineering Council of Canada (06019). Dr. Millar was the recipient of Post-doctoral Fellowships from the Heart and Stroke/Richard Lewar Centre of Excellence, the Heart and Stroke Foundation of Canada, and the Canadian Institutes of Health Research. Dr. Floras holds the Canada Research Chair in Integrative Cardiovascular Biology.

\section{CONFLICT OF INTEREST}

The authors have no conflicts of interest to report. 


\section{REFERENCES}

Callister, R., Ng, A.V., Seals, D.R. 1994. Arm muscle sympathetic nerve activity during preparation for and initiation of leg-cycling exercise in humans. J. Appl. Physiol. 77, 14031410.

Correia, L.C.L., Lakatta, E.G., O'Connor, F.C., Becker, L.C., Clulow, J.F., Townsend. S. et al. 2002. Attenuated cardiovascular reserve during prolonged submaximal exercise in healthy older subjects. J. Am. Coll. Cardiol. 40, 1290-1297.

Crisafulli, A., Marongiu, E., Ogoh, S. 2015. Cardiovascular reflexes activity and their interaction during exercise. Biomed. Res. Int. 2015, 1-10.

Davy, K.P. Seals, D.R. 1994. Total blood volume in healthy young and older men. J. Appl. Physiol. 76, 2069-2062.

Davy, K.P., Seals, D.R., Tanaka, H. 1998. Augmented cardiopulmonary and integrative sympathetic baroreflexes but attenuated peripheral vasoconstriction with age. Hypertension 32, 298-304.

Dinenno, F.A., Seals, D.R., DeSouza, C.A., Tanaka, H. 2001. Age-related decreases in basal limb blood flow in humans: time course, determinants and habitual exercise effects. $J$. Physiol. 531, 573-579. 
Doherty, C.J., Incognito, A.V., Notay, K., Burns, M.J., Slysz, J.T., Seed, J.D. et al. 2018. Muscle sympathetic nerve responses to passive and active one-legged cycling: insights into the contributions of central command. Am. J. Physiol. (Heart Circ. Physiol. ) 314, H3-H10.

Ebert, T.J., Morgan ,B.J., Barney, J.A., Denahan, T., Smith, J.J. 1992. The effects of aging on baroreflex regulation of sympathetic activity in humans. Am..J. Physiol. 263, H798-H803.

Ferrari, A., Radaelli, A., Centola, M. 2003. Aging and the cardiovascular system. J. Appl. Physiol. 95, 2591-2597.

Fisher, J.P., Kim, A., Young, C.N., Fadel, P.J. 2010. Carotid baroreflex control of arterial blood pressure at rest and during dynamic exercise in aging humans. Am. J. Physiol. (Regulatory Integrative Comp. Physiol.) 299, R1241-R1247.

Fleg, J.L., Schulman, S., O'Connor, F., Becker, L,C., Gerstenblith, G., Clulow, J.F. et al. 1994. Effects of acute $\beta$-adrenergic receptor blockade on age-associated changes in cardiovascular performance during dynamic exercise. Circ. 90, 2333-2341.

Franklin, S.S., Gustin, W., Wong, N.D., Larson, M.G., Weber, M.A., Kannel, W.B. et al. 1997. Hemodynamic patterns in age-related changes in blood pressure. The Framingham Heart Study. Circ. 96, 308-315.

Frontera, W.R., Hughes, V.A., Fielding, R.A., Fiatarone, M.A., Evans, W.J., Roubenoff, R. 2000. Aging of skeletal muscle: a 12-yr longitudinal study. J. Appl. Physiol. 88, 1321-1326. 
Greaney, J.L., Schwartz, C.E., Edwards, D.G., Fadel, P.J., Farquhar, W.B. 2013. The neural interactiobetween the arterial baroreflex and muscle metaboreflex is preserved in older men. Exp. Physiol. 98, 1422-1431.

Hart, E.C., Joyner, M.J., Wallin, B.G., Johnson, C.P., Curry, T.B., Eisenach, J.H. et al. 2009. Age-related differences in the sympathetic-hemodynamic balance in men. Hypertension 54, 127-133.

Hart, E.C., Wallin, B.G., Barnes, J.N., Joyner, M.J., Charkoudian, N. 2015. Sympathetic nerve activity and peripheral vasodilator capacity in young and older men. Am. J. Physiol. (Heart Circ. Physiol. ) 306, H904-H909.

Houssiere, A., Najem, B., Pathak, A., Xhaet, O., Naeije, R., van de Borne, P. 2006. Chemoreflex and metaboreflex responses to static hypoxic exercise in aging humans. Med. Sci. Sports. Exerc. 38, 305-312.

Ichinose, M., Saito, M., Fujii, N., Ogawa, T., Hayashi, K., Kondo, N, et al. 2008. Modulation of the control of muscle sympathetic nerve activity during incremental leg cycling. J. Physiol. 586, 2753-2766.

Jones, N.L., Makrides, L., Hitchcock, C., Chychar, T., McCartney, N. 1985. Normal standards for an incremental progressive cycle ergometer test. Am. Rev. Resp. Dis. 131, 700708. 
Katayama, K., Ishida, K., Iwamoto, E., Iemitsu, M., Koike, T., Saito, M. 2011. Hypoxia auments muscle sympathetic neural response to leg cycling. Am. J. Physiol. (Regulatory Integrative Comp. Physiol. ) 301, R456-R464.

Katayama, K., Ishida, K., Saito, M., Koike, T., Hirasawa, K., Ogoh. S, 2014. Enhanced muscle pump during mild dynamic leg exercise inhibits sympathetic vasomotor outflow. Physiol. Reports, 2, e12070.

Lalande, S., Sawicki, C.P., Baker, J.R., Shoemaker, J.K. 2014. Effect of age on the hemodynamic and sympathetic responses at the onset of isometric handgrip exercise. J. Appl. Physiol. 116, 222-227.

Mark, A.L., Victor, R.G., Nerhed, C., Wallin, B.G. 1985. Microneurographic studies of the mechanisms of sympathetic nerve responses to static exercise in humans. Circ. Res. 57, 461469.

Markel, T.A., Daley III, J.C., Hogeman, C.S., Herr, M.D., Khan, M.H., Gray, K.S. et al. 2003. Aging and the exercise pressor reflex in humans. Circ. 107, 675-678.

Matsukawa, T., Sugiyama, Y., Mano, T. 1996. Age-related changes in baroreflex control of heart rate and sympathetic nerve activity in healthy humans. J. Autonom. Nerv. Syst. 60, 209212.

Millar, P.J., Murai, H., Floras J.S. 2015. Paradoxical muscle sympathetic reflex activation in human heart failure. Circ. 131, 459-468. 
Monahan, K.D. 2007. Effect of aging on baroreflex function in humans. Am. J. Physiol. (Regulatory Integrative Comp. Physiol.) 293, R3-R12.

Ng, A.V., Callister, R., Johnson, D.G., Seals, D.R. 1994. Sympathetic neural reactivity to stress does not increase with age in healthy humans. Am. J. Physiol. 267 (Heart and Circ..36), H344-H353.

Noble, B.J., Borg, G.A.V., Jacobs, I., Ruggaro, C., Kaiser, P. 1983. A category-ratio perceived exertion scale: relationship to blood and muscle lactates and heart rate. Med. Sci. Sports Exerc. 15, 523-528.

Notarius, C.F., Millar, P.J., Murai, H., Morris, B.L., Marzolini, S., Oh, P. et al. 2015. Divergent muscle sympathetic responses to dynamic leg exercise in heart failure and agematched healthy subjects. J. Physiol. 593, 715-722.

Olsen, H. Lanne, T. 1998. Reduced venous compliance in lower limbs of aging humans and its importance for capacitance function. Am. J. Physiol. (Heart Circ. Physiol.) 44, H878H886.

Proctor, D.N. Parker, B.A. 2006. Vasodilation and vascular control in contracting muscle of the aging human. Microcirculation 13, 315-327.

Ray, C.A., Rea, R.F., Clary, M.P., Mark, A.L. 1993. Muscle sympathetic nerve responses to dynamic one-legged exercise: effect of body posture. Am. J. Physiol. (Heart Circ .Physiol.) 264, H1-H7. 
Redfield, M.M., Jacobson, S.J., Borlaug, B.A., Rodeheffer, R.J., Kass, D.A. 2005. Age- and gender-related ventricular-vascular stiffening. A community-based study. Circ. 112, 22542262.

Rudas, L., Crossman, A.A., Morillo, C.A., Halliwill, J.R., Tahvanainen, K.U., Eckberg, D.L. 1999. Human sympathetic and vagal baroreflex responses to sequential nitroprusside and phenylephrine. Am. J. Physiol. 276, H1691-H1698.

Saito, M. Mano, T. 1991. Exercise mode affects muscle sympathetic nerve responsiveness. Jpn. J. Physiol. 41, 143-151.

Saito, M., Tsukanaka, A., Yanagihara, D., Mano. T. 1993. Muscle sympathetic nerve responses to graded leg cycling. J. Appl. Physiol. 75, 663-667.

Shi, X., Gallagher, K.M., Welsh-O'Connor, R.M., Foresman, B.H. 1996. Arterial and cardiopulmonary baroreflexes in 60- to 69- vs. 18- to 36-yr-old humans. J. Appl. Physiol. 80, 1903-1910.

Sidhu, S.K., Weavil, J.C., Venturelli, M., Rossman, M.J., Gmelch, B.S., Bledsoe, A.D. et al. 2015. Aging alters muscle reflex control of autonomic cardiovascular responses to rhythmic contractions in humans. Am. J. Physiol. (Heart Circ. Physiol. ) 309, H1479-H1489.

Studinger, P., Goldstein, R., Taylor, J.A. 2009. Age- and fitness-related alterations in vascular sympathetic control. J. Physiol. 587.9, 2049-2057. 
Sundlof ,G. Wallin, B.G. 1978. Human muscle nerve sympathetic activity at rest.

Relationship to blood pressure and age. J. Physiol. 274, 621-637.

Tanaka, H., Davy, K.P., Seals, D.R. 1999. Cardiopulmonary baroreflex inhibition of sympathetic activity is preserved with age in healthy humans. J. Physiol. 515.1, 249-254.

Tanaka, H., Dinenno, F.A., Monahan, K.D., Clevenger, C.M., DeSouza, C.A., Seals, D.R. 2000. Aging, habitual exercise, and dynamic arterial compliance. Circ. 102, 1270-1275.

Valbo, A.B., Hagbarth, K.E., Wallin, B.G. 2004. Microneurography: how the technique developed and its role in the investigation of the sympathetic nervous system. J. Appl. Physiol. 96, 1262-1269.

Victor, R.G., Seals, D.R., Mark, A.L. 1987. Differential control of heart rate and sympathetic nerve activity during dynamic exercise. J. Clin. Invest. 79, 508-516.

World Health Organization. Definition of an older or elderly person. [online] 2013. Geneva, Switzerland, World Health Organization. 8-4-2017. Available from: http://www.who.int/healthinfo/survey/ageingdefnolder/en/index.html. 


\section{Figure Legends}

Figure 1: Representative 30 second traces of MSNA and HR in a young and middle-aged subject during the second minute of pre-cycle, 0 load cycle and moderate load cycle. MSNA, muscle sympathetic nerve activity; HR, heart rate in beats per minute.

Figure 2: Change in heart rate (HR), systolic (SBP) and diastolic (DBP) blood pressure from baseline during moderate 1-leg cycling in middle-aged (open bars $\mathrm{n}=12$ for BP and 18 for HR) and young (closed bars $\mathrm{n}=18)$ subjects. The mean change in $\mathrm{HR}$ is lower $(+, \mathrm{P}=0.03)$, whereas the change in SBP is significantly higher in the middle-aged vs younger group (*, $\mathrm{P}=0.02)$ with no difference in $\mathrm{DBP}$ between groups $(\mathrm{P}=0.21)$.

Figure 3: A: MSNA burst incidence decreases progressively during 1-leg cycling, more in middle-aged (open bars, $n=18$ ) than in young subjects (closed bars, $n=18$ ) (group effect $\mathrm{P}=0.01$; exercise intensity effect $\mathrm{P}=0.04) . * \mathrm{P}=0.03$ vs young subjects. MSNA, muscle sympathetic nerve activity; hb, heart beats. B: MSNA burst frequency decreases to a similar extent in both cohorts (group effect $\mathrm{P}=0.25$ ) with no intensity effect $(\mathrm{P}=0.87)$ or interaction $(\mathrm{P}=0.33)$. 
Table 1.Physical Characteristics and Resting Data.

\begin{tabular}{|c|c|c|}
\hline Variable & Young group & Middle-aged group \\
\hline Number (women) & $18(6)$ & $18(6)$ \\
\hline Age (years) & $22.7 \pm 0.7$ & $56.6 \pm 1.7 *$ \\
\hline Height (cm) & $172.4 \pm 2.2$ & $172.2 \pm 2.7$ \\
\hline Body Weight (kg) & $67.1 \pm 2.8$ & $74.4 \pm 3.7$ \\
\hline BMI $\left(\mathrm{kg} / \mathrm{m}^{2}\right)$ & $22.4 \pm 0.6$ & $25.1 \pm 1.0+$ \\
\hline Heart Rate (bpm) & $62 \pm 2$ & $64 \pm 3$ \\
\hline Systolic Blood Pressure (mmHg) & $112 \pm 5$ & $117 \pm 3$ \\
\hline Diastolic Blood Pressure & $63 \pm 1$ & $68 \pm 2+$ \\
\hline MSNA (bursts/min) & $27.3 \pm 0.9$ & $44.0 \pm 2.0 *$ \\
\hline MSNA (bursts/100 hb) & $44.7 \pm 1.5$ & $70.0 \pm 3.6^{*}$ \\
\hline VO2peak (L/min) & $3.3 \pm 0.2$ & $2.3 \pm 0.2 * *$ \\
\hline VO2peak(ml/kg·min $\left.{ }^{-1}\right)$ & $48.5 \pm 1.8$ & $30.6 \pm 2.2 *$ \\
\hline VO2peak (\% predicted) & $114.7 \pm 5.8$ & $110.3 \pm 6.5$ \\
\hline Peak Work Rate (watts) & $232 \pm 16$ & $144 \pm 17+$ \\
\hline
\end{tabular}

Table 1. Mean \pm SE. *, $\mathrm{P}<0.001$; vs. young group; **, $\mathrm{P}<0.01$; vs. young group; + , P<0.05; BMI, body mass index; MSNA, muscle sympathetic nerve activity; hb, heart beat; VO2, oxygen uptake. 
Young Subject

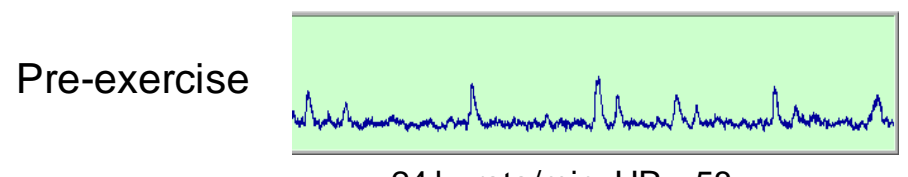

24 bursts $/$ min HR= 58

0 load cycle

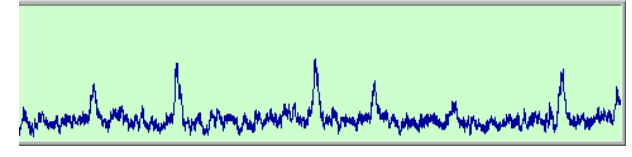

19 bursts $/ \mathrm{min} H R=65$

moderate load cycle

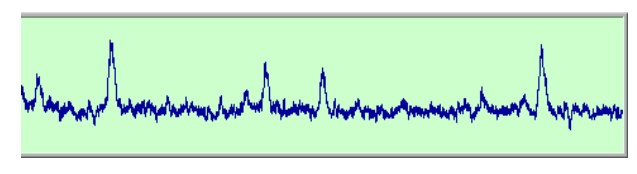

17 bursts $/$ min HR= 62
Middle-aged Subject

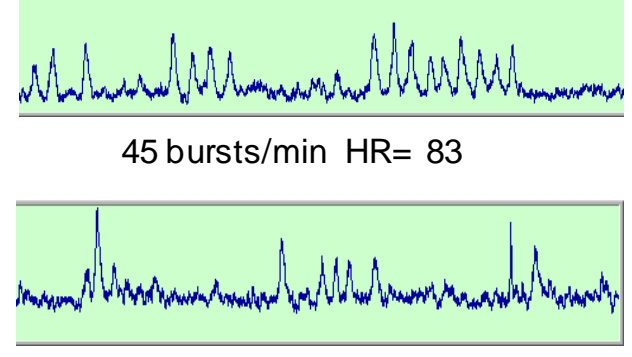

28 bursts $/$ min $H R=89$

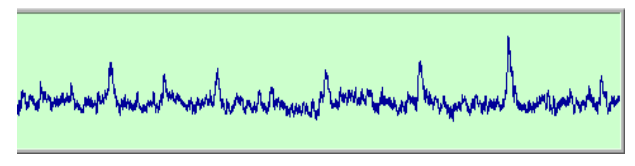

29 bursts $/$ min $H R=94$

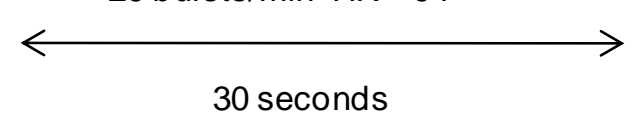




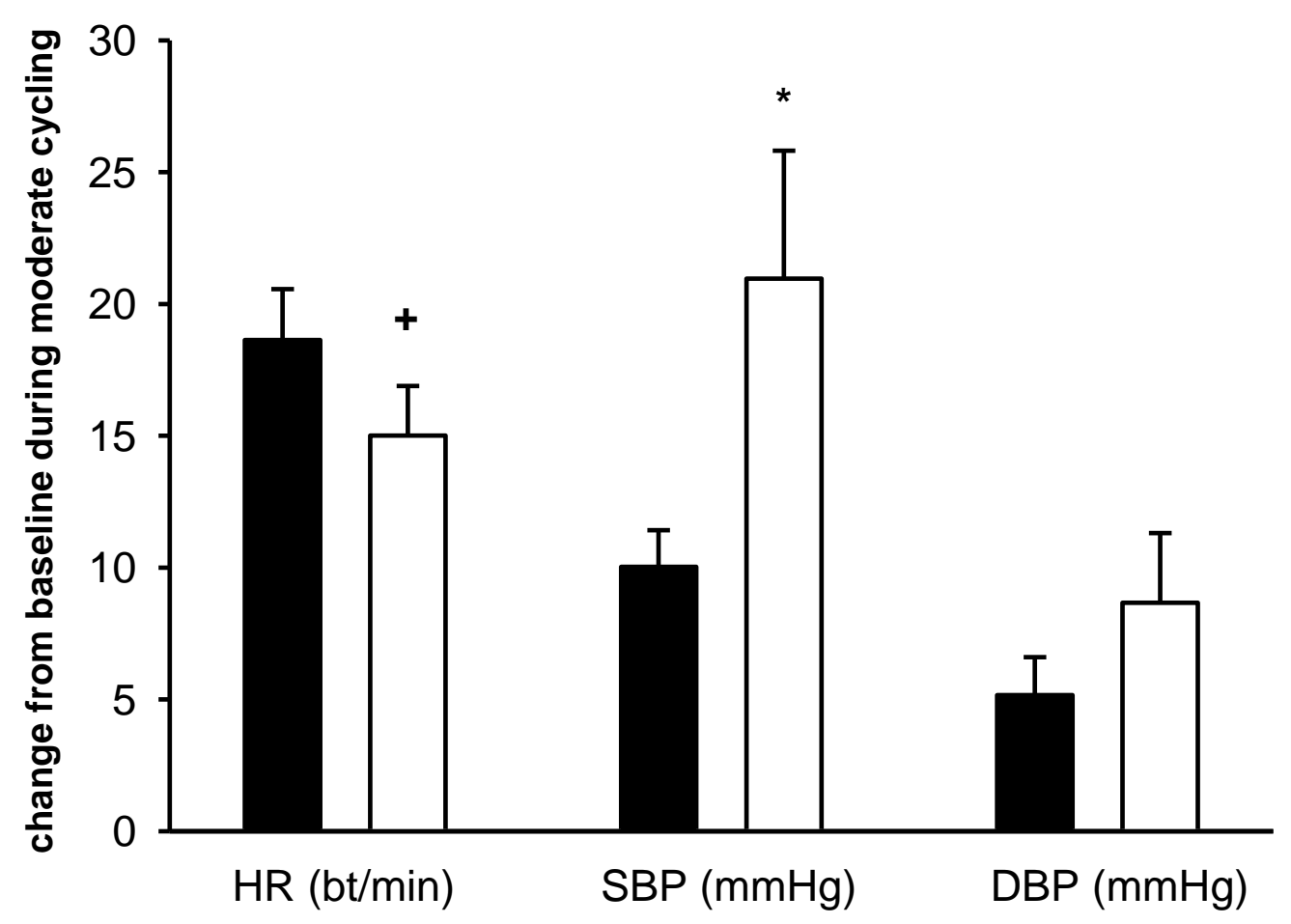


